\title{
On Self-Approaching and Increasing-Chord Drawings of 3-Connected Planar Graphs
}

\author{
Martin Nöllenburg, Roman Prutkin, and Ignaz Rutter \\ Institute of Theoretical Informatics, Karlsruhe Institute of Technology, Germany
}

\begin{abstract}
An st-path in a drawing of a graph is self-approaching if during a traversal of the corresponding curve from $s$ to any point $t^{\prime}$ on the curve the distance to $t^{\prime}$ is non-increasing. A path has increasing chords if it is self-approaching in both directions. A drawing is self-approaching (increasing-chord) if any pair of vertices is connected by a self-approaching (increasing-chord) path.

We study self-approaching and increasing-chord drawings of triangulations and 3 -connected planar graphs. We show that in the Euclidean plane, triangulations admit increasing-chord drawings, and for planar 3-trees we can ensure planarity. Moreover, we give a binary cactus that does not admit a self-approaching drawing. Finally, we show that 3-connected planar graphs admit increasing-chord drawings in the hyperbolic plane and characterize the trees that admit such drawings.
\end{abstract}

\section{Introduction}

Finding a path between two vertices is one of the most fundamental tasks users want to solve when considering graph drawings. Empirical studies have shown that users perform better in path-finding tasks if the drawings exhibit a strong geodesic-path tendency [10,17]. Not surprisingly, graph drawings in which a path with certain properties exists between every pair of vertices have become a popular research topic. Over the last years a number of different drawing conventions implementing the notion of strong geodesic-path tendency have been suggested, namely greedy drawings [18], (strongly) monotone drawings [2], and self-approaching and increasing-chord drawings [1]. Note that throughout this paper, all drawings are straight-line and vertices are mapped to distinct points.

The notion of greedy drawings came first and was introduced by Rao et al. [18]. Motivated by greedy routing schemes, e.g., for sensor networks, one seeks a drawing, where for every pair of vertices $s$ and $t$ there exists an $s t$-path, along which the distances to $t$ decrease in every vertex. This ensures that greedily sending a message to a vertex that is closer to the destination guarantees delivery. Papadimitriou and Ratajczak conjectured that every 3-connected planar graph admits a greedy embedding into the Euclidean plane [16]. This conjecture has been proved independently by Leighton and Moitra [13] and Angelini et al. [5]. Kleinberg [12] showed that every connected graph has a greedy drawing in the hyperbolic plane. Eppstein and Goodrich [7] showed how to construct such an embedding, in which the coordinates of each vertex are represented using only $O(\log n)$ bits, and Goodrich and Strash [9] provided a corresponding succinct representation for greedy embeddings of 3-connected planar graphs in $\mathbb{R}^{2}$. Angelini et al. [3] showed that some graphs require exponential area for a greedy drawing

C. Duncan and A. Symvonis (Eds.): GD 2014, LNCS 8871, pp. 476487 2014.

(c) Springer-Verlag Berlin Heidelberg 2014 
in $\mathbb{R}^{2}$. Wang and He [21] used a custom distance metric to construct planar, convex and succinct greedy embeddings of 3-connected planar graphs using Schnyder realizers [20]. Nöllenburg and Prutkin [14] characterized trees admitting a Euclidean greedy embedding. However, a number of interesting questions remain open, e.g., whether every 3-connected planar graph admits a planar and convex Euclidean greedy embedding (strong Papadimitriou-Ratajczak conjecture [16]). Regarding planar greedy drawings of triangulations, the only known result is an existential proof by Dhandapani [6].

While getting closer to the destination, a greedy path can make numerous turns and may even look like a spiral, which hardly matches the intuitive notion of geodesic-path tendency. To overcome this, Angelini et al. [2] introduced monotone drawings, where one requires that for every pair of vertices $s$ and $t$ there exists a monotone path, i.e., a path that is monotone with respect to some direction. Ideally, the monotonicity direction should be $\overrightarrow{s t}$. This property is called strong monotonicity. Angelini et al. showed that biconnected planar graphs admit monotone drawings [2] and that plane graphs admit monotone drawings with few bends [4]. The existence of strongly monotone planar drawings remains open, even for triangulations.

Both greedy and monotone paths may have arbitrarily large detour, i.e., the ratio of the path length and the distance of the endpoints can, in general, not be bounded by a constant. Motivated by this fact, Alamdari et al. [1] recently initiated the study of self-approaching graph drawings. Self-approaching curves, introduced by Icking [11], are curves where for any point $t^{\prime}$ on the curve, the distance to $t^{\prime}$ decreases continuously while traversing the curve from the start to $t^{\prime}$. Equivalently, a curve is self-approaching if, for any three points $a, b, c$ in this order along the curve, it is $\operatorname{dist}(a, c) \geq \operatorname{dist}(b, c)$, where dist denotes the Euclidean distance. An even stricter requirement are so-called increasing-chord curves, which are curves that are self-approaching in both directions. The name is motivated by the characterization of such curves, which states that a curve has increasing chords if and only if for any four distinct points $a, b, c, d$ in that order, it is $\operatorname{dist}(b, c) \leq \operatorname{dist}(a, d)$. Self-approaching curves have detour at most 5.333 [11] and increasing-chord curves have detour at most 2.094 [19]. Alamdari et al. [1] studied the problem of recognizing whether a given graph drawing is self-approaching as well as connecting given points to a self-approaching drawing. They also gave a complete characterization of trees admitting self-approaching drawings.

We note that every increasing chord drawing is self-approaching and strongly monotone [1]. The converse is not true. A self-approaching drawing is greedy, but not necesserily monotone, and a greedy drawing is generally neither self-approaching nor monotone. For trees, the notions of self-approaching and increasing-chord drawing coincide.

Contribution. We obtain the following results on constructing self-approaching or increasing-chord drawings.

1. We show that every triangulation has an increasing-chord drawing (answering an open question of Alamdari et al. [1]) and construct a binary cactus that does not admit a self-approaching drawing (Sect. 3). The latter is a notable difference to greedy drawings since both constructions of greedy drawings for 3-connected planar graphs [5, 13] essentially show that every binary cactus has a greedy drawing. 
2. We show how to construct plane increasing-chord drawings for planar 3-trees (a special class of triangulations) using Schnyder realizers (Sect. 4). To the best of our knowledge, this is the first construction for this graph class, even for greedy and strongly monotone plane drawings, which addresses an open question of Angelini et al. [2].

3 . We show that, similarly to the greedy case, the hyperbolic plane $\mathbb{H}^{2}$ allows representing a broader class of graphs than $\mathbb{R}^{2}$ (Sect. 5). We prove that a tree has a selfapproaching or increasing-chord drawing in $\mathbb{H}^{2}$ if and only if it either has maximum degree 3 or is a subdivision of $K_{1,4}$ (this is not the case in $\mathbb{R}^{2}$; see the characterization by Alamdari et al. [1]), implying every 3-connected planar graph has an increasingchord drawing. We also show how to construct planar increasing-chord drawings of binary cactuses in $\mathbb{H}^{2}$.

\section{Preliminaries}

For points $a, b, c, d \in \mathbb{R}^{2}$, let $\operatorname{ray}(a, b)$ denote the ray with origin $a$ and direction $\overrightarrow{a b}$ and $\operatorname{ray}(a, \overrightarrow{b c})$ the ray with origin $a$ and direction $\overrightarrow{b c}$. Let $\operatorname{dir}(a b)$ be the vector $\overrightarrow{a b}$ normalized to unit length. Let $\angle(\overrightarrow{a b}, \overrightarrow{c d})$ denote the smaller angle formed by the two vectors

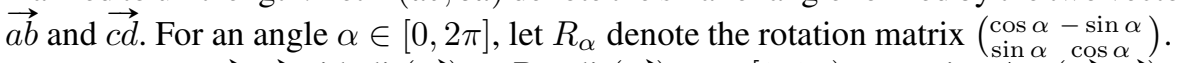

For vectors $\overrightarrow{v_{1}}, \overrightarrow{v_{2}}$ with $\operatorname{dir}\left(\overrightarrow{v_{2}}\right)=R_{\alpha} \cdot \operatorname{dir}\left(\overrightarrow{v_{1}}\right), \alpha \in[0,2 \pi)$, we write $\angle_{\mathrm{ccw}}\left(\overrightarrow{v_{1}}, \overrightarrow{v_{2}}\right):=$ $\alpha$. Further, let $\left[\overrightarrow{v_{1}}, \overrightarrow{v_{2}}\right]$ denote the cone of directions $\left\{\vec{v} \mid \operatorname{dir}(\vec{v})=R_{\beta} \cdot \operatorname{dir}\left(\overrightarrow{v_{1}}\right), \beta \in\right.$ $[0, \alpha]\}$. Let $\left|\left[\overrightarrow{v_{1}}, \overrightarrow{v_{2}}\right]\right|:=\alpha$ be its size. For a set of directions $D$, let $\bar{D}$ denote a minimum cone of directions containing $D$, and let $|D|=|\bar{D}|$. Note that if $|D|<180^{\circ}, \bar{D}$ is unique.

We reuse some notation from the work of Alamdari et al. [1]. For points $p, q \in$ $\mathbb{R}^{2}, p \neq q$, let $l_{p q}^{+}$denote the halfplane not containing $p$ bounded by the line through $q$ orthogonal to the segment $p q$. A piecewise-smooth curve is self-approaching if and only if for each point $a$ on the curve, the line perpendicular to the curve at $a$ does not intersect the curve at a later point [11]. This leads to the following characterization of self-approaching paths.

Fact 1 (Corollary 2 in [1]). Let $\rho=\left(v_{1}, v_{2}, \ldots, v_{k}\right)$ be a directed path embedded in $\mathbb{R}^{2}$ with straight-line segments. Then, $\rho$ is self-approaching if and only if for all $1 \leq i<j \leq k$, the point $v_{j}$ lies in $l_{v_{i} v_{i+1}}^{+}$.

We shall denote the reverse of a path $\rho$ by $\rho^{-1}$. Let $\rho=$ $\left(v_{1}, v_{2}, \ldots, v_{k}\right)$ be a self-approaching path. Define front $(\rho)=$ $\bigcap_{i=1}^{k-1} l_{v_{i} v_{i+1}}^{+}$, see also Fig. 1. Using Fact 1, we can decide whether a concatenation of two paths is self-approaching.

Fact 2. Let $\rho_{1}=\left(v_{1}, \ldots, v_{k}\right)$ and $\rho_{2}=\left(v_{k}, v_{k+1}, \ldots, v_{m}\right)$ be self-approaching paths. The path $\rho_{1} . \rho_{2}:=\left(v_{1}, \ldots, v_{k}, v_{k+1}\right.$, $\left.\ldots, v_{m}\right)$ is self-approaching if and only if $\rho_{2} \subseteq \operatorname{front}\left(\rho_{1}\right)$.

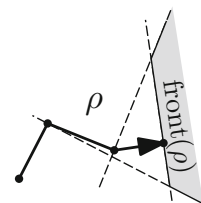

Fig. 1. self-approaching path $\rho$ and front $(\rho)$

A path $\rho$ has increasing chords if for any points $a, b, c, d$ in this order along $\rho$, it is $\operatorname{dist}(b, c) \leq \operatorname{dist}(a, d)$. A path has increasing chords if and only if it is self-approaching in both directions. The following result is easy to see. 
Lemma 1. Let $\rho=\left(v_{1}, \ldots, v_{k}\right)$ be a path such that for any $i<j, i, j \in\{1, \ldots, k-1\}$, it is $\angle\left(\overrightarrow{v_{i} v_{i+1}}, \overrightarrow{v_{j} v_{j+1}}\right) \leq 90^{\circ}$. Then, $\rho$ has increasing chords.

Let $G=(V, E)$ be a connected graph. A separating $k$-set is a set of $k$ vertices whose removal disconnects the graph. A vertex forming a separating 1 -set is called cutvertex. A graph is c-connected if it does not admit a separating $k$-set with $k \leq c-1 ; 2$-connected graphs are also called biconnected. A connected graph is biconnected if and only if it does not contain a cutvertex. A block is a maximal biconnected subgraph. The blockcutvertex tree (or $B C$-tree) $T_{G}$ of $G$ has a $B$-node for each block of $G$, a $C$-node for each cutvertex of $G$ and, for each block $\nu$ containing a cutvertex $v$, an edge between the corresponding $B$ - and $C$-node. We associate $B$-nodes with their corresponding blocks and $C$-nodes with their corresponding cutvertices.

The following notation follows the work of Angelini et al. [5]. Let $T_{G}$ be rooted at some block $\nu$ containing a non-cutvertex (such a block $\nu$ always exists). For each block $\mu \neq \nu$, let $\pi(\mu)$ denote the parent block of $\mu$, i.e., the grandparent of $\mu$ in $T_{G}$. Let $\pi^{2}(\mu)$ denote the parent block of $\pi(\mu)$ and, generally, $\pi^{i+1}(\mu)$ the parent block of $\pi^{i}(\mu)$. Further, we define the root $r(\mu)$ of $\mu$ as the cutvertex contained in both $\mu$ and $\pi(\mu)$. Note that $r(\mu)$ is the parent of $\mu$ in $T_{G}$. In addition, for the root node $\nu$ of $T_{G}$, we define $r(\nu)$ to be some non-cutvertex of $\nu$. Let depth ${ }_{B}(\mu)$ denote the number of $B$-nodes on the $\nu \mu$-path in $T_{G}$ minus 1 , and let depth $C(r(\mu))=\operatorname{depth}_{B}(\mu)$. If $\mu$ is a leaf of $T_{G}$, we call it a leaf block.

If every block of $G$ is outerplanar, $G$ is called a cactus. In a binary cactus every cutvertex is part of exactly two blocks. For a binary cactus $G$ with a block $\mu$ containing a cutvertex $v$, let $G_{\mu}^{v}$ denote the maximal connected subgraph containing $v$ but no other vertex of $\mu$. We say that $G_{\mu}^{v}$ is a subcactus of $G$.

A cactus is triangulated if each of its blocks is internally triangulated. A triangular fan with vertices $V_{t}=\left\{v_{0}, v_{1}, \ldots, v_{k}\right\}$ and root $v_{0}$ is a graph on $V_{t}$ with edges $v_{i} v_{i+1}$, $i=1, \ldots, k-1$, as well as $v_{0} v_{i}, i=1, \ldots, k$. Let us consider a special kind of triangulated cactuses, each of whose blocks $\mu$ is either an edge or a triangular fan with root $r(\mu)$. We call such a cactus downward-triangulated and every edge of a block $\mu$ incident to $r(\mu)$ a downward edge.

For a fixed straight-line drawing of a binary cactus $G$, we define sets $U(G)=$ $\{\overrightarrow{r(\mu) v} \mid \mu$ is a block of $G$ containing $v, v \neq r(\mu)\}$ and $D(G)=\{\overrightarrow{u v} \mid \overrightarrow{v u} \in U(G)\}$, i.e. the sets of upward and downward directions of $G$.

\section{Graphs with Self-Approaching Drawings}

A natural approach to construct (not necessarily plane) self-approaching drawings is to construct a self-approaching drawing of a spanning subgraph. For instance, to draw a graph $G$ containing a Hamiltonian path $H$ with increasing chords, we simply draw $H$ consecutively on a line. In this section, we consider 3-connected planar graphs and the special case of triangulations, which addresses an open question of Alamdari et al. [1]. These graphs are known to have a spanning binary cactus [5, 13]. Angelini et al. [5] showed that every triangulation has a spanning downward-triangulated binary cactus. 


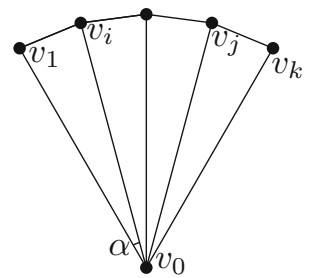

(a)

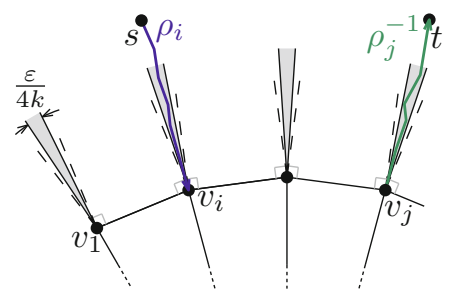

(b)

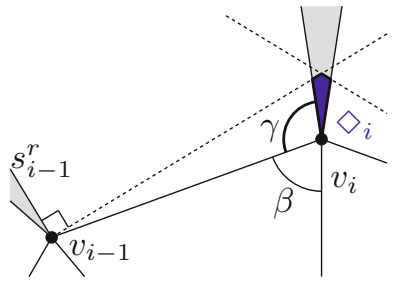

(c)

Fig. 2. Drawing a triangulated binary cactus with increasing chords inductively. The drawings $\Gamma_{i, \varepsilon^{\prime}}$ of the subcactuses, $\varepsilon^{\prime}=\frac{\varepsilon}{4 k}$, are contained inside the gray cones. It is $\beta=90^{\circ}-\varepsilon^{\prime}$, $\gamma=90^{\circ}+\varepsilon^{\prime} / 2$.

\subsection{Increasing-Chord Drawings of Triangulations}

We show that every downward-triangulated binary cactus has an increasing-chord drawing. The construction is similar to the one of the greedy drawings of binary cactuses in the two proofs of the Papadimitriou-Ratajczak conjecture [5,13]. Our proof is by induction on the height of the BC-tree. We show that $G$ can be drawn such that all downward edges are almost vertical and the remaining edges almost horizontal. For vertices $s, t$, an $s t$-path with increasing chords goes downwards to some block $\mu$, then sideways to another cutvertex of $\mu$ and, finally, upwards to $t$. Let $\overrightarrow{e_{1}}, \overrightarrow{e_{2}}$ be vectors $(1,0)^{\top},(0,1)^{\top}$.

Theorem 1. Let $G=(V, E)$ be a downward-triangulated binary cactus. For any $0^{\circ}<$ $\varepsilon<90^{\circ}$, there exists an increasing-chord drawing $\Gamma_{\varepsilon}$ of $G$, such that for each vertex $v$ contained in some block $\mu, v \neq r(\mu)$, the angle formed by $\overrightarrow{r(\mu) v}$ and $\overrightarrow{e_{2}}$ is at most $\frac{\varepsilon}{2}$.

Proof. Let $G$ be rooted at block $\nu$. As our base case, let $\nu=G$ be a triangular fan with vertices $v_{0}, v_{1}, \ldots, v_{k}$ and root $v_{0}=r(\nu)$. We draw $v_{0}$ at the origin and distribute $v_{1}, \ldots, v_{k}$ on the unit circle, such that $\angle\left(\overrightarrow{e_{2}}, \overrightarrow{v_{0} v_{1}}\right)=k \alpha / 2$ and $\angle\left(\overrightarrow{v_{0} v_{i}}, \overrightarrow{v_{0} v_{i+1}}\right)=\alpha$, $\alpha=\varepsilon / k$; see Fig. 2a. By Lemma1, path $\left(v_{1}, \ldots, v_{k}\right)$ has increasing chords.

Now let $G$ have multiple blocks. We draw the root block $\nu, v_{0}=r(\nu)$, as in the previous case, but with $\alpha=\frac{\varepsilon}{2 k}$. Then, for each $i=1, \ldots, k$, we choose $\varepsilon^{\prime}=\frac{\varepsilon}{4 k}$ and draw the subcactus $G_{i}$ rooted at $v_{i}$ inductively, such that the corresponding drawing $\Gamma_{i, \varepsilon^{\prime}}$ is aligned at $\overrightarrow{v_{0} v_{i}}$ instead of $\overrightarrow{e_{2}}$; see Fig $2 \mathrm{~b}$. Note that $\varepsilon^{\prime}$ is the angle of the cones (gray) containing $\Gamma_{i, \varepsilon^{\prime}}$. Obviously, all downward edges form angles at most $\frac{\varepsilon}{2}$ with $\overrightarrow{e_{2}}$.

We must be able to reach any $t$ in any $G_{j}$ from any $s$ in any $G_{i}$ via an increasingchord path $\rho$. To achieve this, we make sure that no normal on a downward edge of $G_{i}$ crosses the drawing of $G_{j}, j \neq i$. Let $\Lambda_{i}$ be the cone with apex $v_{i}$ and angle $\varepsilon^{\prime}$ aligned with $\overrightarrow{v_{0} v_{i}}, v_{0} \notin \Lambda_{i}$ (gray regions in Fig. 2b). Let $s_{i}^{l}$ and $s_{i}^{r}$ be the left and right boundary rays of $\Lambda_{i}$ with respect to $\overrightarrow{v_{0} v_{i}}$, and $h_{i}^{l}$, $h_{i}^{r}$ the halfplanes with boundaries containing $v_{i}$ and orthogonal to $s_{i}^{l}$ and $s_{i}^{r}$ respectively, such that $v_{0} \in h_{i}^{l} \cap h_{i}^{r}$. Define $\diamond_{i}=\Lambda_{i} \cap$ $h_{i-1}^{r} \cap h_{i+1}^{l}$ (thin blue quadrangle in Fig. 2c), and analogously $\diamond_{j}$ for $j \neq i$. It holds $\diamond_{j} \subseteq h_{i}^{r} \cap h_{i}^{l}$ for each $i \neq j$. We now scale each drawing $\Gamma_{i, \varepsilon^{\prime}}$ such that it is contained in $\diamond_{i}$. In particular, for any downward edge $u v$ in $\Gamma_{i, \varepsilon^{\prime}}$, we have $\Gamma_{j, \varepsilon^{\prime}} \subseteq \diamond_{j} \subseteq l_{u v}^{+}$for $j \neq i$. We claim that the resulting drawing of $G$ is an increasing-chord drawing. 
Consider vertices $s, t$ of $G$. If $s$ and $t$ are contained in the same subgraph $G_{i}$, an increasing-chord st-path in $G_{i}$ exists by induction. If $s$ is in $G_{i}$ and $t$ is $v_{0}$, let $\rho_{i}$ be the $s v_{i}$-path in $G_{i}$ that uses only downward edges. By Lemma1 path $\rho_{i}$ is increasing-chord and remains so after adding edge $v_{i} v_{0}$.

Finally, assume $t$ is in $G_{j}$ with $j \neq i$. Let $\rho_{j}$ be the $t v_{j}$-path in $G_{j}$ that uses only downward edges. Due to the choice of $\varepsilon^{\prime}, h_{i}^{r} \cap h_{i}^{l} \subseteq$ front $\left(\rho_{i}\right)$ contains $v_{1}, \ldots, v_{k}$ in its interior. Consider the path $\rho^{\prime}=\left(v_{i}, v_{i+1}, \ldots, v_{j}\right)$. It is self-approaching by Lemma 1 , also, $\rho^{\prime} \subseteq \operatorname{front}\left(\rho_{i}\right)$ and $\rho_{j} \subseteq$ front $\left(\rho^{\prime}\right)$. It also holds $\rho_{j} \subseteq \diamond_{j} \subseteq$ front $\left(\rho_{i}\right)$. Fact 2]lets us concatenate $\rho_{i}, \rho^{\prime}$ and $\rho_{j}^{-1}$ to a self-approaching path. By a symmetric argument, it is also self-approaching in the opposite direction and, thus, is increasing-chord.

Since every triangulation has a spanning downward-triangulated binary cactus [5], this implies that planar triangulations admit increasing-chord drawings.

Corollary 1. Every planar triangulation admits an increasing-chord drawing.

\subsection{Non-triangulated Cactuses}

The above construction fails if the blocks are not triangular fans since we now cannot just use downward edges to reach the common ancestor block. Consider the family of rooted binary cactuses $G_{n}=\left(V_{n}, E_{n}\right)$ defined as follows. Graph $G_{0}$ is a single 4-cycle, where an arbitrary vertex is designated as the root. For $n \geq 1$, consider two disjoint copies of $G_{n-1}$ with roots $a_{0}$ and $c_{0}$. We create $G_{n}$ by adding new vertices $r_{0}$ and $b_{0}$ both adjacent to $a_{0}$ and $c_{0}$; see Fig. 3a. For the new block $\nu$ containing $r_{0}, a_{0}, b_{0}, c_{0}$, we set $r(\nu)=r_{0}$. We select $r_{0}$ as the root of $G_{n}$ and $\nu$ as its root block. For a block $\mu_{i}$ with root $r_{i}$, let $a_{i}, b_{i}, c_{i}$ be its remaining vertices, such that $b_{i} r_{i} \notin E_{n}$. For a given drawing, due to the symmetry of $G_{n}$, we can rename the vertices $a_{i}$ and $c_{i}$ such that $\angle_{\mathrm{ccw}}\left(\overrightarrow{r_{i} c_{i}}, \overrightarrow{r_{i} a_{i}}\right) \leq 180^{\circ}$. We now prove the following negative result.

Theorem 2. For $n \geq 9, G_{n}$ has no self-approaching drawing.

The outline of the proof is as follows. We show that every self-approaching drawing $\Gamma$ of $G_{9}$ contains a self-approaching drawing of $G_{3}$ with the following properties.

1. If $\mu_{i}$ is contained in the subcactus rooted at $c_{j}$, each self-approaching $b_{i} a_{j}$-path uses edge $b_{i} a_{i}$, and analogously for the symmetric case; see Lemma 5 .

2. Each block is drawn significantly smaller than its parent block; see Lemma 6(i).

3. If the descendants of block $\mu$ form subcactuses $G_{k}$ with $k \geq 2$ on both sides, the parent block of $\mu$ must be drawn smaller than $\mu$; see Lemma 6 (ii).

Obviously, the second and third conditions are contradictory. The following lemmas will be used to show that the drawings of certain blocks must be relatively thin, i.e., their downward edges have similar directions; see the full version for the omitted proofs [15].

Lemma 2. For cactus $G=(V, E)$ and $s, t \in V$, consider cutvertices $v_{1}, \ldots, v_{k}$ lying on any st-path in $G$ in this order. Then, the path $\left(s, v_{1}, \ldots, v_{k}, t\right)$ is drawn greedily, i.e., each of its subpaths is greedy. In particular, ray $\left(v_{1}, s\right)$ and ray $\left(v_{k}, t\right)$ diverge.

Obviously, this divergence property also holds for a self-approaching drawing of any cactus. From now on, we consider a fixed self-approaching drawing $\Gamma$ of $G_{9}$. For 


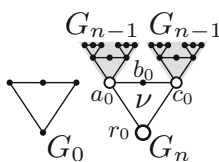

(a)

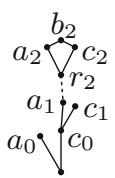

(b)

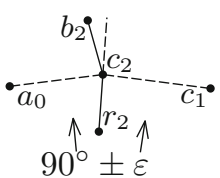

(c)

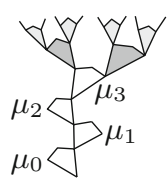

(d)

Fig. 3. (a) cactuses $G_{n}$; (b) (c) construction for Lemma 5 (d) subcactus $G_{5}$ providing the contradiction in the proof of Theorem 2

a block $\mu$ of $G_{9}$ with root $r=r(\mu)$, we write $G^{r}$ for $\left(G_{9}\right)_{\mu}^{r}$, i.e., the binary cactus subgraph of $G_{9}$ rooted at $r$. We write $U^{r}$ for the set of directions of the upward edges of $G^{r}$ and define $I^{r}=\overline{U^{r}}$. Using Lemma 2, we can show that vectors in $U^{a_{i}} \cup U^{c_{i}}$ have the following circular order: first vectors in $U^{a_{i}}$, then vectors in $U^{c_{i}}$. It follows easily: $\min \left\{\left|I^{a_{i}}\right|,\left|I^{c_{i}}\right|\right\}<\left|I^{r_{i}}\right| / 2$. Thus, we can provide a bound for the smallest of the cones of a subcactus depending on the depth of its root.

Lemma 3. Every self-approaching drawing of $G_{9}$ contains a cutvertex $\bar{r}$ with $\operatorname{depth}_{C}(\bar{r})=4$ and $\left|I^{\bar{r}}\right|<22.5^{\circ}$.

Let $\bar{r}$ be a cutvertex from Lemma 3 in the fixed drawing, and let $\varepsilon:=\left|I^{\bar{r}}\right|$. Then, $G^{\bar{r}}$ is isomorphic to $G_{6}$. From now on, we only consider non-leaf blocks $\mu_{i}$ and vertices $r_{i}, a_{i}, b_{i}, c_{i}$ in $G^{\bar{r}}$. We shall sometimes name the points $a$ instead of $a_{i}$ etc. for convenience. We assume $\angle\left(\overrightarrow{e_{2}}, \overrightarrow{r a}\right), \angle\left(\overrightarrow{e_{2}}, \overrightarrow{r c}\right)<\varepsilon / 2$. The following lemma is proved using basic trigonometric arguments.

Lemma 4. It holds: (i) $\angle a b c \geq 90^{\circ}$; (ii) $G^{a} \subseteq l_{b a}^{+}, G^{c} \subseteq l_{b c}^{+}$; (iii) $\angle b a r \leq 90^{\circ}+\varepsilon$, $\angle b c r \leq 90^{\circ}+\varepsilon$. (iv) For vertices $u$ in $G^{a}$, v in $G^{c}$ of degree 4 it is $\angle\left(\overrightarrow{u v}, \overrightarrow{e_{1}}\right) \leq \varepsilon / 2$

We can now describe block angles at $a_{i}, c_{i}$ more precisely and characterize certain self-approaching paths in $G^{\bar{r}}$. We show that a self-approaching path from $b_{i}$ downwards and to the left, i.e., to an ancestor block $\mu_{j}$ of $\mu_{i}$, such that $\mu_{i}$ is in $G^{c_{j}}$, must use $a_{i}$. Similarly, a self-approaching path downwards and to the right must use $c_{i}$. Since for several ancestor blocks of $\mu_{i}$ the roots lie on both of these two kinds of paths, we can bound the area containing them and show that it is relatively small. This implies that the ancestor blocks are small as well, providing a contradiction.

Lemma 5. Consider non-leaf blocks $\mu_{0}, \mu_{1}, \mu_{2}$, such that $r\left(\mu_{1}\right)=c_{0}$ and $\mu_{2}$ in $G^{a_{1}}$; see Fig. $3 b$. (i) It is $\angle r_{2} a_{2} b_{2}, \angle r_{2} c_{2} b_{2} \in\left[90^{\circ}, 90^{\circ}+\varepsilon\right], b_{2}$ lies to the right of $\operatorname{ray}\left(r_{2}, a_{2}\right)$ and to the left of $\operatorname{ray}\left(r_{2}, c_{2}\right)$. (ii) Each self-approaching $b_{2} a_{0}$-path uses $a_{2}$; each selfapproaching $b_{2} c_{1}$-path uses $c_{2}$.

Proof. (i) Assume $\angle r_{2} a_{2} b_{2}<90^{\circ}$. Then, all self-approaching $b_{2} a_{0}$ and $b_{2} c_{1}$-paths must use $c_{2}$. By Lemma 4 (iv), the lines through $a_{0} c_{2}$ and $c_{2} c_{1}$ are "almost horizontal", i.e., $\angle\left(\overrightarrow{a_{0} c_{2}}, \overrightarrow{e_{1}}\right), \angle\left(\overrightarrow{c_{2} c_{1}}, \overrightarrow{e_{1}}\right) \leq \varepsilon / 2$. Since $r_{2} c_{2}$ is "almost vertical", $r_{2}$ must lie below these lines and it is $\angle a_{0} c_{2} r_{2}, \angle c_{1} c_{2} r_{2} \in\left[90^{\circ}-\varepsilon, 90^{\circ}+\varepsilon\right]$; see Fig. 3c. First, let $b_{2}$ lie to the left of ray $\left(r_{2}, c_{2}\right)$. Then, $b_{2}$ is above $a_{0} c_{2}$, and it is $\angle r_{2} c_{2} b_{2}=\angle a_{0} c_{2} r_{2}+\angle a_{0} c_{2} b_{2} \geq$ 


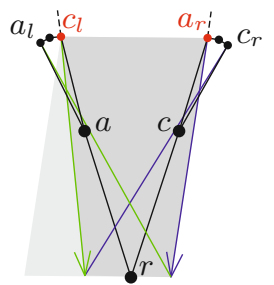

(a)

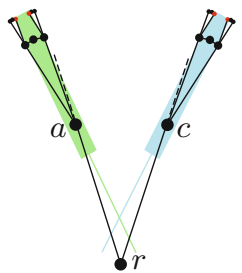

(b)

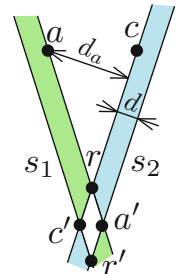

(c)

Fig. 4. Showing the contradiction in Theorem 2

$\left(90^{\circ}-\varepsilon\right)+90^{\circ}$. Now let $b_{2}$ lie to the right of ray $\left(r_{2}, c_{2}\right)$. Then, $b_{2}$ is above $c_{2} c_{1}$, and it is $\angle r_{2} c_{2} b_{2}=\angle c_{1} c_{2} r_{2}+\angle c_{1} c_{2} b_{2} \geq\left(90^{\circ}-\varepsilon\right)+90^{\circ}$. Since $\varepsilon<22.5^{\circ}$, this contradicts Lemma 4(iii). Similarly, $\angle r_{2} c_{2} b_{2} \geq 90^{\circ}$. Thus, by Lemma 4 (iii), $\angle r_{2} c_{2} b_{2}, \angle r_{2} c_{2} b_{2} \in$ $\left[90^{\circ}, 90^{\circ}+\varepsilon\right]$. Since $\angle a_{2} b_{2} c_{2} \geq 90^{\circ}, b_{2}$ lies to the right of $\operatorname{ray}\left(r_{2}, a_{2}\right)$ and to the left of ray $\left(r_{2}, c_{2}\right)$. (If $b_{2}$ lies to the left of both rays, it is $\angle a_{2} b_{2} c_{2}=\angle\left(\overrightarrow{a_{2} b_{2}}, \overrightarrow{c_{2} b_{2}}\right) \leq 2 \varepsilon<$ $90^{\circ}$.) (ii) Similarly, if a self-approaching $b_{2} a_{0}$-path uses $c_{2}$ instead of $a_{2}$, it is $\angle r_{2} c_{2} b_{2} \geq$ $180^{\circ}-\varepsilon$. The last part follows analogously.

From now on, let $\mu_{0}$ be the root block of $G^{\bar{r}}$ and $\mu_{1}, \mu_{2}, \mu_{3}$ its descendants such that $r\left(\mu_{1}\right)=c_{0}, r\left(\mu_{2}\right)=a_{1}, r\left(\mu_{3}\right) \in\left\{a_{2}, c_{2}\right\}$; see Fig. 3d. Light gray blocks are the subject of Lemma 6(i), which shows that several ancestor roots lie inside a cone with a small angle. Dark gray blocks are the subject of Lemma 6(ii), which considers the intersection of the cones corresponding to a pair of sibling blocks and shows that some of their ancestor roots lie inside a narrow strip; see Fig. 4a for a sketch.

Lemma 6. Let $\mu$ be a block in $G^{c_{2}}$ with vertices $a, b, c, r(\mu)$. (i) Let $\mu$ have depth 5 in $G^{\bar{r}}$. Then, the cone $l_{b a}^{+} \cap l_{b c}^{+}$contains $r(\mu), r(\pi(\mu)), r\left(\pi^{2}(\mu)\right)$ and $r\left(\pi^{3}(\mu)\right)$. (ii) Let $\mu$ have depth 4 in $G^{\bar{r}}$. There exist $u$ in $G^{a}$ and $v$ in $G^{c}$ of degree 4 and a strip $S$ containing $r(\mu), r(\pi(\mu)), r\left(\pi^{2}(\mu)\right)=r\left(\mu_{2}\right)$, such that $u$ and $v$ lie on the different boundaries of $S$.

Again, we consider two siblings and the intersection of their corresponding strips, which forms a small diamond containing the root of the ancestor block; see Fig. 4b, 4c,

Lemma 7. Consider block $\mu=\mu_{3}$ containing $r=r(\mu), a, b$, c, and let $r_{\pi}:=r\left(\pi\left(\mu_{3}\right)\right)$. It holds: (i) $\left|r_{\pi} r\right| \leq \frac{(1+2 \tan \varepsilon)(\tan \varepsilon)^{2}}{\cos \varepsilon}(|r a|+|r c|)$; (ii) $|r a|,|r c| \leq\left|r r_{\pi}\right|(\tan \varepsilon)^{2}$.

For $\varepsilon \leq 22.5^{\circ}$, the two claims of Lemma7 7 contradict each other. This concludes the proof of Theorem 2 .

\section{Planar Increasing-Chord Drawings of 3-Trees}

In this section, we show how to construct planar increasing-chord drawings of 3-trees. We make use of Schnyder labelings [20] and drawings of triangulations based on them. For a plane triangulation $G=(V, E)$ with external vertices $r, g, b$, its Schnyder labeling is an orientation and partition of the interior edges into three trees $T_{r}, T_{g}, T_{b}$ (called 
red, green and blue tree), such that for each internal vertex $v$, its incident edges appear in the following clockwise order: exactly one outgoing red, an arbitrary number of incoming blue, exactly one outgoing green, an arbitrary number of incoming red, exactly one outgoing blue, an arbitrary number of incoming green. Each of the three outer vertices $r, g, b$ serves as the root of the tree in the same color and all its incident interior edges are incoming in the respective color. For $v \in V$, let $R_{v}^{r}$ (the red region of $v$ ) denote the region bounded by the $v g$-path in $T_{g}$, the $v b$-path in $T_{b}$ and the edge $g b$. Let $\left|R_{v}^{r}\right|$ denote the number of the interior faces in $R_{v}^{r}$. The green and blue regions $R_{v}^{g}, R_{v}^{b}$ are defined analogously. Assigning $v$ the coordinates $\left(\left|R_{v}^{r}\right|,\left|R_{v}^{g}\right|,\left|R_{v}^{b}\right|\right) \in \mathbb{R}^{3}$ results in a plane straight-line drawing of $G$ in the plane $\left\{x=\left(x_{1}, x_{2}, x_{3}\right) \mid x_{1}+x_{2}+x_{3}=f-1\right\}$ called Schnyder drawing. Here, $f$ denotes the number of faces of $G$. For a thorough introduction to this topic, see the book of Felsner [8].

For $\alpha, \beta \in\left[0^{\circ}, 360^{\circ}\right]$, let $[\alpha, \beta]$ denote the corresponding counterclockwise cone of directions. We consider drawings satisfying the following constraints.

Definition 1. Let $G=(V, E)$ be a plane triangulation graph with a Schnyder labeling. For $0^{\circ} \leq \alpha \leq 60^{\circ}$, we call an arbitrary planar straight-line drawing of $G \alpha$-Schnyder if for each internal vertex $v \in V$, its outgoing red edge has direction in $\left[90^{\circ}-\frac{\alpha}{2}, 90^{\circ}+\frac{\alpha}{2}\right]$, blue in $\left[210^{\circ}-\frac{\alpha}{2}, 210^{\circ}+\frac{\alpha}{2}\right]$ and green in $\left[330^{\circ}-\frac{\alpha}{2}, 330^{\circ}+\frac{\alpha}{2}\right]$ (see Fig. $5 a$ ).

According to Def.11 classical Schnyder drawings are $60^{\circ}$-Schnyder. The next lemma shows an interesting connection between $\alpha$-Schnyder and increasing-chord drawings.

Lemma 8. $30^{\circ}$-Schnyder drawings are increasing-chord drawings.

Proof. Let $G=(V, E)$ be a plane triangulation with a given Schnyder labeling and $\Gamma$ a corresponding $30^{\circ}$-Schnyder drawing. Let $r, g, b$ be the red, green and blue external vertex, respectively, and $T_{r}, T_{g}, T_{b}$ the directed trees of the corresponding color.

Consider vertices $s, t \in V$. First, note that monochromatic directed paths in $\Gamma$ have increasing chords by Lemma11 Assume $s$ and $t$ are not connected by such a path. Then, they are both internal and $s$ is contained in one of the regions $R_{t}^{r}, R_{t}^{g}, R_{t}^{b}$. Without loss of generality, we assume $s \in R_{t}^{r}$. The $s r$-path in $T_{r}$ crosses the boundary of $R_{t}^{r}$, and we assume without loss of generality that it crosses the blue boundary of $R_{t}^{r}$ in $u \neq t$; see Fig. 5b. The other cases are symmetric.

Let $\rho_{r}$ be the $s u$-path in $T_{r}$ and $\rho_{b}$ the $t u$-path in $T_{b}$; see Fig. 5c On the one hand, the direction of a line orthogonal to a segment of $\rho_{r}$ is in $\left[345^{\circ}, 15^{\circ}\right] \cup\left[165^{\circ}, 195^{\circ}\right]$. On the other hand, $\rho_{b}$ is contained in a cone $\left[15^{\circ}, 45^{\circ}\right]$ with apex $u$. Thus, $\rho_{b}^{-1} \subseteq \operatorname{front}\left(\rho_{r}\right)$, and $\rho_{r} \cdot \rho_{b}^{-1}$ is self-approaching by Fact 2 By a symmetric argument it is also self-approaching in the other direction, and hence has increasing chords.

Planar 3-trees are the graphs obtained from a triangle by repeatedly choosing a (triangular) face $f$, inserting a new vertex $v$ into $f$, and connecting $v$ to each vertex of $f$.

Lemma 9. Planar 3-trees have $\alpha$-Schnyder drawings for any $0^{\circ}<\alpha \leq 60^{\circ}$.

Proof. We describe a recursive construction of an $\alpha$-Schnyder drawing of a planar 3tree. We start with an equilateral triangle and put a vertex $v$ in its center. Then, we align 


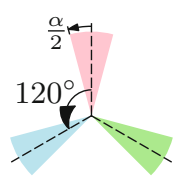

(a)

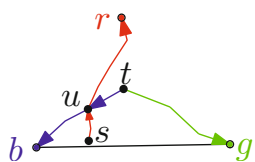

(b)

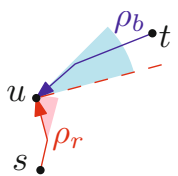

(c)

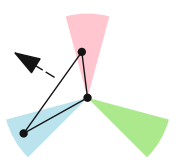

(d)

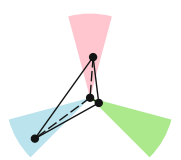

(e)

Fig. 5. (a) (c) $30^{\circ}$-Schnyder drawings are increasing-chord; (d) (e) special case of planar 3-trees.

the pattern from Fig. 5a at $v$. For the induction step, consider a triangular face $x y z$ and assume that the pattern is centered at one of its vertices, say $x$, such that the other two vertices are in the interiors of two distinct cones; see Fig. 5d, It is now possible to move the pattern inside the triangle slightly, such that the same holds for all three vertices $x, y, z$; see Fig. 5e We insert the new vertex at the center of the pattern and again get the situation as in Fig. 5d,

Lemmas 8 and 9 provide a constructive proof for the following theorem.

Theorem 3. Every planar 3-tree has a planar increasing-chord drawing.

\section{Self-Approaching Drawings in the Hyperbolic Plane}

Kleinberg [12] showed that any tree can be drawn greedily in the hyperbolic plane $\mathbb{H}^{2}$. This is not the case in $\mathbb{R}^{2}$. Thus, $\mathbb{H}^{2}$ is more powerful than $\mathbb{R}^{2}$ in this regard. Since selfapproaching drawings are closely related to greedy drawings, it is natural to investigate the existence of self-approaching drawings in $\mathbb{H}^{2}$.

We shall use the Poincaré disk model for $\mathbb{H}^{2}$, in which $\mathbb{H}^{2}$ is represented by the unit disk $D=\left\{x \in \mathbb{R}^{2}:|x|<1\right\}$ and lines are represented by circular arcs orthogonal to the boundary of $D$. For an introduction to the Poincaré disk model, see e.g. Kleinberg [12] and the references therein.

First, let us consider a tree $T=(V, E)$. A drawing of $T$ in $\mathbb{R}^{2}$ is self-approaching if and only if no normal on an edge of $T$ in any point crosses another edge [1]. The same condition holds in $\mathbb{H}^{2}$; see full version for the proof [15]. According to the characterization by Alamdari et al. [1], some binary trees have no self-approaching drawings in $\mathbb{R}^{2}$. We show that this is no longer the case in $\mathbb{H}^{2}$.

Theorem 4. Let $T=(V, E)$ be a tree, such that each node of $T$ has degree either 1 or 3. Then, $T$ has a self-approaching drawing in $\mathbb{H}^{2}$, in which every arc has the same hyperbolic length and every pair of incident arcs forms an angle of $120^{\circ}$.

Proof. For convenience, we subdivide each edge of $T$ once. We shall show that both pieces are collinear in the resulting drawing $\Gamma$ and have the same hyperbolic length.

First, consider a regular hexagon $\square=p_{0} p_{1} p_{2} p_{3} p_{4} p_{5}$ centered at the origin $o$ of $D$; see Fig. 6a. In $\mathbb{H}^{2}$, it can have angles smaller than $120^{\circ}$. We choose them to be $90^{\circ}$ (any angle between $0^{\circ}$ and $90^{\circ}$ would work). Next, we draw a $K_{1,3}$ with center $v_{0}$ in $o$ and the leaves $v_{1}, v_{2}, v_{3}$ in the middle of the arcs $p_{0} p_{1}, p_{2} p_{3}, p_{4} p_{5}$ respectively. 


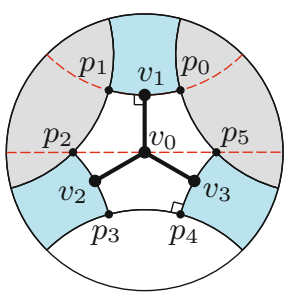

(a)

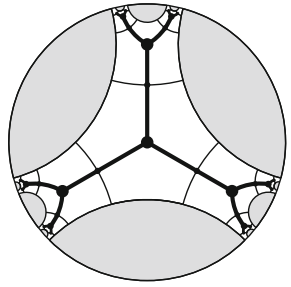

(b)

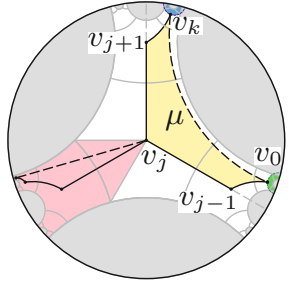

(c)

Fig. 6. Constructing increasing-chord drawings of binary trees and cactuses in $\mathbb{H}^{2}$

For each such building block of the drawing consisting of a $K_{1,3}$ inside a regular hexagon with $90^{\circ}$ angles, we add its copy mirrored at an arc of the hexagon containing a leaf node of the tree constructed so far. For example, in the first iteration, we add three copies of $\square$ mirrored at $p_{0} p_{1}, p_{2} p_{3}$ and $p_{4} p_{5}$, respectively, and the corresponding inscribed $K_{1,3}$ subtrees. The construction after two iterations is shown in Fig. 6b. This process can be continued infinitely to construct a drawing $\Gamma_{\infty}$ of the infinite binary tree. However, we stop after we have completed $\Gamma$ for the tree $T$.

We now show that $\Gamma_{\infty}$ (and thus also $\Gamma$ ) has the desired properties. Due to isometries and the aforementioned sufficient condition, it suffices to consider edge $e=v_{0} v_{1}$ and show that a normal on $e$ does not cross $\Gamma_{\infty}$ in another point. To see this, consider Fig. 6a Due to the choice of the angles of $\square$, all the other hexagonal tiles of $\Gamma_{\infty}$ are contained in one of the three blue quadrangular regions $\square_{i}:=l_{v_{0} v_{i}}^{+} \backslash\left(l_{v_{i} p_{2 i-1}}^{+} \cup l_{v_{i} p_{2 i-2}}^{+}\right), i=1,2,3$. Thus, the regions $l_{v_{1} p_{1}}^{+}$and $l_{v_{1} p_{0}}^{+}$(gray) contain no point of $\Gamma_{\infty}$. Therefore, since each normal on $v_{0} v_{1}$ is contained in the "slab" $D \backslash\left(l_{v_{0} v_{1}}^{+} \cup l_{v_{1} v_{0}}^{+}\right)$bounded by the diameter through $p_{2}, p_{5}$ and the line through $p_{0}, p_{1}$ (dashed) and is parallel to both of these lines, it contains no other point of $\Gamma_{\infty}$.

We note that our proof is similar in spirit to the one by Kleinberg [12], who also used tilings of $\mathbb{H}^{2}$ to prove that any tree has a greedy drawing in $\mathbb{H}^{2}$.

As in the Euclidean case, it can be easily shown that if a tree $T$ contains a node $v$ of degree 4, it has a self-approaching drawing in $\mathbb{H}^{2}$ if and only if $T$ is a subdivision of $K_{1,4}$ (apply an isometry, such that $v$ is in the origin of $D$ ). This completely characterizes the trees admitting a self-approaching drawing in $\mathbb{H}^{2}$. Further, it is known that every binary cactus and, therefore, every 3-connected planar graph has a binary spanning tree [5, 13].

Corollary 2. (i) A tree $T$ has an increasing-chord drawing in $\mathbb{H}^{2}$ if and only if $T$ either has maximum degree 3 or is a subdivision of $K_{1,4}$. (ii) Every binary cactus and, therefore, every 3-connected planar graph has an increasing-chord drawing in $\mathbb{H}^{2}$.

Again, note that this is not the case for binary cactuses in $\mathbb{R}^{2}$; see the example in Theorem 2. We use the above construction to produce planar self-approaching drawings of binary cactuses in $\mathbb{H}^{2}$. We show how to choose a spanning tree and angles at vertices of degree 2, such that non-tree edges can be added without introducing crossings; see Fig. 6c for a sketch and the full version [15] for the proof.

Corollary 3. Every binary cactus has a planar increasing-chord drawing in $\mathbb{H}^{2}$. 


\section{References}

1. Alamdari, S., Chan, T.M., Grant, E., Lubiw, A., Pathak, V.: Self-approaching Graphs. In: Didimo, W., Patrignani, M. (eds.) GD 2012. LNCS, vol. 7704, pp. 260-271. Springer, Heidelberg (2013)

2. Angelini, P., Colasante, E., Di Battista, G., Frati, F., Patrignani, M.: Monotone drawings of graphs. J. Graph Algorithms Appl. 16(1), 5-35 (2012)

3. Angelini, P., Di Battista, G., Frati, F.: Succinct greedy drawings do not always exist. Networks 59(3), 267-274 (2012)

4. Angelini, P., Didimo, W., Kobourov, S., Mchedlidze, T., Roselli, V., Symvonis, A., Wismath, S.: Monotone drawings of graphs with fixed embedding. In: Speckmann, B. (ed.) GD 2011. LNCS, vol. 7034, pp. 379-390. Springer, Heidelberg (2012)

5. Angelini, P., Frati, F., Grilli, L.: An algorithm to construct greedy drawings of triangulations. J. Graph Algorithms Appl. 14(1), 19-51 (2010)

6. Dhandapani, R.: Greedy drawings of triangulations. Discrete Comput. Geom. 43, 375-392 (2010)

7. Eppstein, D., Goodrich, M.T.: Succinct greedy geometric routing using hyperbolic geometry. IEEE Trans. Computers 60(11), 1571-1580 (2011)

8. Felsner, S.: Geometric Graphs and Arrangements. Vieweg+Teubner Verlag (2004)

9. Goodrich, M.T., Strash, D.: Succinct greedy geometric routing in the Euclidean plane. In: Dong, Y., Du, D.-Z., Ibarra, O. (eds.) ISAAC 2009. LNCS, vol. 5878, pp. 781-791. Springer, Heidelberg (2009)

10. Huang, W., Eades, P., Hong, S.-H.: A graph reading behavior: Geodesic-path tendency. In: IEEE Pacific Visualization Symposium (PacificVis 2009), pp. 137-144 (2009)

11. Icking, C., Klein, R., Langetepe, E.: Self-approaching curves. Math. Proc. Camb. Phil. Soc. 125, 441-453 (1999)

12. Kleinberg, R.: Geographic routing using hyperbolic space. In: Computer Communications (INFOCOM 2007), pp. 1902-1909. IEEE (2007)

13. Moitra, A., Leighton, T.: Some Results on Greedy Embeddings in Metric Spaces. Discrete Comput. Geom. 44, 686-705 (2010)

14. Nöllenburg, M., Prutkin, R.: Euclidean greedy drawings of trees. In: Bodlaender, H.L., Italiano, G.F. (eds.) ESA 2013. LNCS, vol. 8125, pp. 767-778. Springer, Heidelberg (2013)

15. Nöllenburg, M., Prutkin, R., Rutter, I.: On Self-Approaching and Increasing-Chord Drawings of 3-Connected Planar Graphs. CoRR arXiv:1409.0315 (2014)

16. Papadimitriou, C.H., Ratajczak, D.: On a conjecture related to geometric routing. Theor. Comput. Sci. 344(1), 3-14 (2005)

17. Purchase, H.C., Hamer, J., Nöllenburg, M., Kobourov, S.G.: On the usability of Lombardi graph drawings. In: Didimo, W., Patrignani, M. (eds.) GD 2012. LNCS, vol. 7704, pp. 451462. Springer, Heidelberg (2013)

18. Rao, A., Ratnasamy, S., Papadimitriou, C., Shenker, S., Stoica, I.: Geographic routing without location information. In: Mobile Computing and Networking (MobiCom 2003), pp. 96-108. ACM (2003)

19. Rote, G.: Curves with increasing chords. Math. Proc. Camb. Phil. Soc. 115, 1-12 (1994)

20. Schnyder, W.: Embedding planar graphs on the grid. In: Discrete Algorithms (SODA 1990), pp. 138-148. SIAM (1990)

21. Wang, J.-J., He, X.: Succinct strictly convex greedy drawing of 3-connected plane graphs. Theor. Comput. Sci. 532, 80-90 (2014) 